\title{
Symbolic computation of the Birkhoff normal form in the problem of stability of the triangular libration points
}

\author{
I. I. Shevchenko* \\ Pulkovo Observatory of the Russian Academy of Sciences \\ Pulkovskoje ave. 65/1, St.Petersburg 196140, Russia
}

\begin{abstract}
The problem of stability of the triangular libration points in the planar circular restricted three-body problem is considered. A software package, intended for normalization of autonomous Hamiltonian systems by means of computer algebra, is designed so that normalization problems of high analytical complexity could be solved. It is used to obtain the Birkhoff normal form of the Hamiltonian in the given problem. The normalization is carried out up to the 6th order of expansion of the Hamiltonian in the coordinates and momenta. Analytical expressions for the coefficients of the normal form of the 6th order are derived. Though intermediary expressions occupy gigabytes of the computer memory, the obtained coefficients of the normal form are compact enough for presentation in typographic format. The analogue of the Deprit formula for the stability criterion is derived in the 6th order of normalization. The obtained floating-point numerical values for the normal form coefficients and the stability criterion confirm the results by Markeev (1969) and Coppola and Rand (1989), while the obtained analytical and exact numeric expressions confirm the results by Meyer and Schmidt (1986) and Schmidt (1989). The given computational problem is solved without
\end{abstract}

*E-mail: iis@gao.spb.ru 
constructing a specialized algebraic processor, i.e., the designed computer algebra package has a broad field of applicability.

Keywords: Hamiltonian dynamics; Normal forms; Computer algebra; Circular restricted three-body problem; Lagrange solutions; Triangular libration points.

\section{Introduction}

In 1772 Lagrange [1] discovered a periodic solution of the general threebody problem (TBP), in which the bodies are situated at the apices of an equilateral triangle and each body moves in a Keplerian orbit about the center of mass of the system. In the circular restricted TBP, these periodic solutions correspond to the triangular libration points.

Gascheau [2] found the necessary condition for the stability of the Lagrange periodic solutions in the general TBP:

$$
\frac{\left(m_{1}+m_{2}+m_{3}\right)^{2}}{m_{1} m_{2}+m_{2} m_{3}+m_{1} m_{3}}>27
$$

where $m_{1}, m_{2}, m_{3}$ are the masses of the bodies. In the circular restricted TBP this condition reduces to the inequalities

$$
0<27 \mu(1-\mu)<1
$$

or, equivalently,

$$
0<\mu<\mu^{*}=\frac{9-69^{1 / 2}}{18}=0.0385 \ldots,
$$

where $\mu=m_{2} /\left(m_{1}+m_{2}\right), m_{2}<m_{1}, m_{3}=0$.

Leontovich [3] proved that, in the planar circular restricted TBP, the triangular libration points were stable for all $\mu$ values satisfying condition (2) but a set of values of measure zero. Deprit and Deprit-Bartholomé [4] proved that this exceptional set consisted of only three points. For these three points $\mu_{1}=0.0242 \ldots, \mu_{2}=0.0135 \ldots$, and $\mu_{3}=0.0109 \ldots$ the stability problem remained unsolved at that time.

The $\mu_{1}$ and $\mu_{2}$ values correspond to the resonances 2:1 and 3:1 between the frequencies of the linearized system describing the motion in the neighborhood of the libration points. The $\mu_{3}$ value correspond to a case when a specific algebraic combination of the system frequencies and the coefficients 
of the 4th order normal form of the Hamiltonian of the considered problem is equal to zero. In the general case of an arbitrary $\mu$ value, this expression is usually expressed as a function of the linearized system frequencies, which, in their turn, can be expressed through $\mu$. Henceforth we call this expression, derived by Deprit and Deprit-Bartholomé [4] for the given problem, the Deprit formula. In the notation adopted henceforth, it looks as follows [4]:

$$
D_{4}=\frac{644 \gamma^{4}-541 \gamma^{2}+36}{16\left(4 \gamma^{2}-1\right)\left(25 \gamma^{2}-4\right)}
$$

where $\gamma=\omega_{1} \omega_{2}$, and $\omega_{1}$ and $\omega_{2}$ are the frequencies.

Markeev [5, 6] performed approximate floating point computations of the coefficients of the normal forms for the resonant values of $\mu$, i.e., $\mu_{1}$ and $\mu_{2}$, and for the non-resonant $\mu=\mu_{3}$ case. He proved that the system was unstable in the first two cases, and stable in the third case.

Sokolsky [7] derived an advanced normal form in the critical case, i.e., for the mass ratio $\mu^{*}$, and by evaluating the normal form coefficients in approximate floating point computations proved that the system was formally stable in this case.

Consideration of the problem of stability of the triangular libration points in the planar circular restricted three-body problem was thus completed in the middle of seventies; however, the necessary coefficients of the normal forms were not known in the exact form at that time. Solely their floatingpoint values were available. Their exact numerical representations were obtained later on.

The analytical treatment of the non-resonant $\mu=\mu_{3}$ case is very complicated in what concerns the volume of analytical computation, since attaining of the 6th order of normalization is required. The values of the 6 th order normal form coefficients and the corresponding stability criterion were obtained in the floating-point arithmetic with the help of especially designed computer software by Markeev [5, 8]. Later on, they were calculated also in the floating-point arithmetic by Coppola and Rand [9].

The analytical expression for the stability criterion in the $\mu=\mu_{3}$ case was obtained by Meyer and Schmidt [10] and Schmidt [11]. The exact numerical expression for the stability criterion was calculated by Schmidt [11]. The given computational tasks were solved by means of constructing specialized algebraic processors, in PL/I and in MACSYMA. In [9], the software package in MACSYMA was designed without constructing a specialized algebraic 
processor, but its ability in the given problem was limited solely to computing the coefficients in the floating-point arithmetic.

The exact numeric expressions for the coefficients of the normal forms for the resonances 2:1 and 3:1 $\left(\mu=\mu_{1}\right.$ and $\left.\mu_{2}\right)$ were obtained, to our knowledge, only in the beginning of nineties [12, 13] by means of application of a computer algebra package written in REDUCE. In both resonant cases the exact numeric expressions for the normal form coefficients completely agreed with Markeev's approximate results to their accuracy (the latter results had been stated in [5, 6, 8] with the accuracy of 3-4 significant digits). However, only the expression for the most complicated case, that of the 3:1 resonance, corresponding to the $\mu_{2}$ mass ratio, was published [12, 13], as an example of application of the software package. Note that this resonant value $\mu_{2}=0.0135 \ldots$ is close to the $\mu$ value in the Earth-Moon system, $\mu_{\text {Earth-Moon }}=0.0121 \ldots$

The normal form for the critical mass ratio $\mu^{*}$ was calculated analytically by Schmidt [14] and Bruno and Petrov [15]. A significant (by an order of magnitude) disagreement was evident with Sokolsky's [7] approximate result for a coefficient of the normal form; but the final theoretical inference on the stability in this case remained unchanged, due to the fact that the sign of the coefficient was correct. We should note that one of the reasons for this discrepancy might be a severe loss of accuracy in the cumbersome floatingpoint computations.

Bruno and Petrov [15] accomplished symbolic computations of the normal forms for the 2:1 and 3:1 resonances. The obtained exact numeric expressions for the normal form coefficients completely agreed with Markeev's [5, 6, 8] results to the accuracy of his computations. Bruno and Petrov [15] also performed floating point computations of the coefficients of the normal form for the non-resonant $\mu=\mu_{3}$ case and found agreement with Markeev's [5, 8] results.

In this quite a technical paper, analytical expressions for the non-resonant normal form coefficients and the analogue of the Deprit formula for the stability criterion in the 6th order of normalization are derived. A software package, intended for normalization of autonomous Hamiltonian systems, is designed in the language of the Maple computer algebra system [16] and applied to the given problem. The obtained floating-point numerical values for the normal form coefficients and the stability criterion confirm the results by Markeev [5, 6, 8] and Coppola and Rand [9], while the obtained analytical and exact numerical expressions confirm the results by Meyer and 
Schmidt [10] and Schmidt [11]. The given computational problem is solved without constructing a specialized algebraic processor, i.e., the designed computer algebra package has a broad field of applicability.

\section{Normal forms}

Analysis of the local properties of the solutions of a Hamiltonian system can be accomplished by the method of normal forms. The method allows one to find the approximate general solutions in the neighborhood of the points of equilibria as well as to analyze the motion stability. The complete procedure of normalization of an autonomous Hamiltonian system in the neighborhood of a point of equilibrium implies expansion of the Hamiltonian in the power series of the canonical variables, linear normalization of the system, and its nonlinear normalization.

We assume that the roots of the characteristic equation of the linearized autonomous Hamiltonian system are exclusively imaginary, and that the resonances up to the second order inclusive are absent, i.e., there are no zero or equal frequencies of the system. Then the quadratic part of the Hamiltonian can be transformed to the normal form [17, 18, 8]:

$$
H_{2}=\frac{1}{2} \sum_{k=1}^{N} \lambda_{k}\left(q_{k}^{2}+p_{k}^{2}\right),
$$

where $q_{k}$ and $p_{k}$ are the coordinate and momentum variables, $N$ is the number of degrees of freedom, $\lambda_{k}=\delta_{k} \omega_{k}, \delta_{k}= \pm 1$. The positive constants $\omega_{k}=\left|\lambda_{k}\right|$ are the frequencies of the linearized system at the point of equilibrium.

In the non-resonant case (i.e., when the frequencies $\omega_{k}$ are incommensurate with each other up to the resonance of order $M$ ), via the "polar" canonical change of variables

$$
q_{k}=\left(2 r_{k}\right)^{1 / 2} \sin \varphi_{k}, p_{k}=\left(2 r_{k}\right)^{1 / 2} \cos \varphi_{k},
$$

where $k=1, \ldots, N$, the Hamiltonian normalized up to an arbitrary order $M$ of the Taylor expansion is expressed through the variables $r_{k}$ only. The Birkhoff normal form [17, 18, 8] of order $M$ is given by the following expression: 


$$
K^{(M)}=\sum_{k=1}^{N} \lambda_{k} r_{k}+\sum_{n=2}^{[M / 2]} \sum_{\ell_{1}+\ldots+\ell_{N}=n} c_{\ell_{1}, \ldots, \ell_{N}} r_{1}^{\ell_{1}} \ldots r_{N}^{\ell_{N}},
$$

where $[M / 2]$ is the round part of $M / 2$. The coefficients $c_{\ell_{1}, \ldots, \ell_{N}}$ are invariants of the Hamiltonian with respect to the choice of the normalizing transformation.

The Hamiltonian normalized up to the order $M$ has the form $K=K^{(M)}+$ $h^{(\geq M+1)}$, where $h^{(\geq M+1)}$ are the terms of degree $M+1$ and higher in relation to the variables $q_{k}, p_{k}$, or, equivalently, $h^{(\geq M+1)}$ are the terms of degree $[M / 2]+1$ and higher in relation to the variables $r_{k}$. When resonances are present, the normal forms contain also angle variables in resonant combinations [8, 19].

\section{Algorithms and computation methods}

In the beginning of nineties the specialized computer algebra package "Norma" [12, 20, 13] was developed for performing all analytical procedures necessary for normalization of autonomous Hamiltonian systems in analytical form. The programs were written in the language of the REDUCE [21] computer algebra system. The package allows one to accomplish expansion of the initial Hamiltonian in power series, linear normalization of the system, and its nonlinear normalization.

It is assumed that the roots of the characteristic equation of the linearized system are exclusively imaginary, and that the resonances up to the second order inclusive are absent, i.e., there are no zero or equal frequencies of the system. Then the quadratic part of the Hamiltonian is reduced to the normal form (5).

The basis of the algorithm of linear normalization consists in analytical calculation of the eigenvectors of the matrix of the linearized system. In the package programs, they are computed by means of calculation of the matrix adjoint to the characteristic one. This approach provides the capability to find the simplest (in the analytical sense) version of the formulae of linear normalization.

The nonlinear normalization is accomplished by the Deprit-Hori method [22, 23] in the Mersman modification [24]. The number of degrees of freedom and the order of normalization are arbitrary. The coefficients of the initial Hamiltonian can be set numeric (in exact representation) or symbolic. 
The formulae of the direct normalizing canonical transformation of the variables and those of the inverse transformation are computed by means of programs implementing the procedure of the Lie transformation. The program of nonlinear normalization computes the normalized Hamiltonian and the generating function of the normalizing transformation. The normalizing transformation of the canonical variables is calculated as the Lie transformation with the generator equal to the newly found generating function.

Thus by means of the programs of the described package one can find the Taylor expansion of the Hamiltonian, then normalize the quadratic part of this expansion (i.e., accomplish linear normalization of the system), and, finally, normalize the expansion up to the required order (i.e., accomplish nonlinear normalization). This is the general scheme. Detailed descriptions of the "Norma" algorithms and programs are given in [12, 20, 13.

The application of the "Norma" package to the analysis of the motion near the triangular libration points in the planar circular RTBP provided an opportunity to obtain the analytically simplest formulae of linear normalization [20] and the resonant normal forms up to the 4th order of the Hamiltonian expansion [12, 13] (see Introduction).

Application [25] of the normalization package "NF" written in the Maple computer algebra system to the given problem produced practically the same results. The "Norma" and "NF" packages did not allow one to perform normalization in the non-resonant case of the given problem in the 5th and 6th orders of the Hamiltonian expansion due to high memory consumption: the increase in memory load at critical stages of the computation was too sharp, because the time uniformity in memory consumption had not yet been algorithmically accomplished. Such uniformity can be achieved mainly by means of employing the "reinitialization" procedure (see below), pertinent choice of routines of simplification of analytical expressions, excessive parametrization, and combinations of these methods.

Attaining the 6th order of normalization in the given problem is the subject of the current paper. A new software package, "NP" ("Normalization Package"), has been developed that allows one to solve normalization problems of high complexity level. The "NP" programs have been written in the language of the Maple computer algebra system [16]. All computations have been performed in the Maple system release 9.5.

The "NP" programs are intended for normalization of a Hamiltonian system in the same assumptions on the kind of the Hamiltonian as in the case of the "Norma" package. As in the latter case, the Deprit-Hori method [22, 
23, 24, 19, based on the Lie transformations, is applied for carrying out the nonlinear normalization.

The employed normalization procedure is non-recurrent: the formulae of normalization are programmed explicitly in each order of normalization. This approach abandons the necessity to store the voluminous arrays of auxiliary analytical expressions. The calculation of the auxiliary polynomials $G_{i}$, whose monomial analysis determines the homogeneous components $K_{i}$ of the normalized Hamiltonian $K$ and the homogeneous components $S_{i}$ of the generating function $S$ (see [12, 13]), and whose analytical computation involves a major part of the total memory consumption, is accomplished by the following formulae, given in [19]:

$$
\begin{aligned}
G_{3}= & H_{3}, \\
G_{4}= & H_{4}+\frac{1}{2} D^{1}\left(H_{3}+K_{3}\right), \\
G_{5}= & H_{5}+\frac{1}{2} D^{2}\left(H_{3}+K_{3}\right)+\frac{1}{2} D^{1}\left[H_{4}+K_{4}+\frac{1}{6} D^{1}\left(H_{3}-K_{3}\right)\right], \\
G_{6}= & H_{6}+\frac{1}{2} D^{3}\left(H_{3}+K_{3}\right)+\frac{1}{2} D^{2}\left[H_{4}+K_{4}+\frac{1}{6} D^{1}\left(H_{3}-K_{3}\right)\right]+ \\
& +\frac{1}{2} D^{1}\left[H_{5}+K_{5}+\frac{1}{6} D^{1}\left(H_{4}-K_{4}\right)+\frac{1}{6} D^{2}\left(H_{3}-K_{3}\right)\right],
\end{aligned}
$$

where the Lie differential operator with the generator $S$ is defined by the relations $D^{n} f=D^{1} D^{n-1} f, D^{1} f=\{f, S\}(n=2,3, \ldots) ;\{f, S\}$ denotes the Poisson brackets of the functions $f$ and $S ; H_{i}$ are the homogeneous components of the linearly normalized original Hamiltonian $H$.

Apart from the tools decreasing the total memory load, an effective method of minimizing the peak memory consumption is to make the memory load more uniform in time. This is achieved by using the reinitialization procedure [26] for the coefficients of the polynomials in work. The use of the reinitialization at key stages of the algorithm allows one to solve computeralgebraic problems of high analytical complexity. The economy of the computer memory is attained due to redistribution of memory load in time. The reinitialization procedure is similar in some way to the "method of telescoping compositions", proposed and used in [9], in particular for calculation of the multiple Poisson brackets.

The general procedure of reinitialization of the coefficients of any polynomial at a memory-consuming step of the algorithm of normalization consists 
in creation of a file containing the coefficients of the given polynomial in analytical form, temporary storing this file, while analytical operations with the given polynomial with the undefined coefficients are carried out, and subsequent resubstitution of the stored expressions for the coefficients in the resulting expression. Only then the monomial simplification of the resulting expression is accomplished. In the package, the reinitialization is performed for the coefficients of the homogeneous components of the Hamiltonian and the generating function directly before the calculation of the polynomials $G_{i}$. Right after the calculation, the resubstitution and the monomial simplification are carried out.

The package consists of three basic parts.

The first part contains programs for obtaining the expansion of the Hamiltonian in Taylor series. The file with the initial data contains a number of degrees of freedom, an order of expansion, and a Hamiltonian as a function of the coordinate and momentum variables, $\vec{q}$ and $\vec{p}$. The procedure carries out expansion of the Hamiltonian in Taylor series with the maximum economy of memory, by an algorithm described in [20]. A file containing the expansion of the Hamiltonian is created.

The second part of the package is intended for transforming the Hamiltonian to the linearly normalized form. However, the package does not contain a procedure of linear normalization itself. Linear normalization can be carried out with the help of the "Norma" package. Using the "Norma" output file containing the linear normalizing transformation, the substitutions in all components of the expansion of the initial Hamiltonian are carried out and subsequent monomial simplification is accomplished. A file with the linearly normalized Hamiltonian is created.

The third part of the package contains procedures necessary for nonlinear normalization of the Hamiltonian up to the 6th order in the coordinate and momenta variables. The library contains the following procedures: (a) procedures for calculating the Poisson brackets employing various ways of simplification of the expressions; these ways can be varied depending on the considered problem; (b) a procedure for reinitialization of the coefficients of polynomials; (c) procedures for allocation of the monomials for inclusion in the generating function, the non-resonant and resonant parts of the Hamiltonian; (d) a basic procedure of normalization in the given order. This part of the package produces a file with the components $S_{i}$ of the generating function and the components $K_{i}$ of the normalized Hamiltonian. 


\section{The normal form coefficients and the ana- logue of the Deprit formula}

Now we apply the procedure of normalization in a study of the problem of stability of the triangular libration points in the planar circular RTBP. Consider the non-resonant case (i.e., the frequencies $\omega_{k}$ are incommensurate with each other to the high enough resonance order) of the motion close to the triangular libration point $L_{4}$ in the planar circular RTBP. The dynamical system has two degrees of freedom. The libration point $L_{4}$ is located at the origin of the rotating reference frame. One of the axes of the frame is directed towards the planet. The derivation of the Hamiltonian system of the differential equations describing the motion is described in detail in [8]. The expansion of the Hamiltonian up to the 6th order has the form [8]:

$$
\begin{aligned}
& H=\frac{1}{2}\left(p_{1}^{2}+p_{2}^{2}\right)+p_{1} q_{2}-q_{1} p_{2}+\frac{1}{8}\left(q_{1}^{2}-8 k q_{1} q_{2}-5 q_{2}^{2}\right)+ \\
& +3^{1 / 2}\left(-\frac{7}{36} k q_{1}^{3}+\frac{3}{16} q_{1}^{2} q_{2}+\frac{11}{12} k q_{1} q_{2}^{2}+\frac{3}{16} q_{2}^{3}\right)+ \\
& +\frac{37}{128} q_{1}^{4}+\frac{25}{24} k q_{1}^{3} q_{2}-\frac{123}{64} q_{1}^{2} q_{2}^{2}-\frac{15}{8} k q_{1} q_{2}^{3}-\frac{3}{128} q_{2}^{4}+ \\
& +3^{1 / 2}\left(\frac{23}{576} k q_{1}^{5}-\frac{285}{286} q_{1}^{4} q_{2}-\frac{215}{288} k q_{1}^{3} q_{2}^{2}+\frac{345}{128} q_{1}^{2} q_{2}^{3}+\frac{555}{576} k q_{1} q_{2}^{4}-\frac{33}{256} q_{2}^{5}\right)- \\
& -\frac{331}{1024} q_{1}^{6}+\frac{49}{128} k q_{1}^{5} q_{2}+\frac{6105}{1024} q_{1}^{4} q_{2}^{2}-\frac{35}{64} k q_{1}^{3} q_{2}^{3}-\frac{7965}{1024} q_{1}^{2} q_{2}^{4}-\frac{119}{128} k q_{1} q_{2}^{5}+\frac{383}{1024} q_{2}^{6},
\end{aligned}
$$

where $q_{i}, p_{i}(i=1,2)$ are the canonical variables (the coordinates and momenta in the chosen frames), and the constant denoted by $k$ is $k=$ $3 \cdot 3^{1 / 2}(1-2 \mu) / 4$. We could as well expand the initial Hamiltonian by means of our package and obtain the identical formula in seconds. The coefficients can be parameterized through one of the frequencies of the linearized system by means of the formulae

$$
\begin{aligned}
k & =\left(23+4\left(\omega_{1}^{2}-\omega_{2}^{2}\right)^{2}\right)^{1 / 2} / 4, \\
\omega_{2} & =\left(1-\omega_{1}^{2}\right)^{1 / 2},
\end{aligned}
$$

which can be easily deduced from basic relations given in [8]. In the procedures of normalization, however, it is usually pertinent to retain an excessive 
parametrization, in order to avoid emergence of cumbersome radicals; see notes on this matter in [10]. This is an important auxiliary tool to minimize the memory load.

We linearly normalize the Hamiltonian and create a file with the initial data for the subsequent nonlinear normalization. The file contains the number of degrees of freedom (equal to two), the order of normalization (equal to six), and the linearly normalized Hamiltonian.

The program of nonlinear normalization produces the normalized Hamiltonian. The 4 th order homogeneous component $K_{4}$ of the obtained normal form $K^{(4)}$ has the form

$$
\begin{aligned}
K_{4}= & -\frac{1}{144} \frac{\omega_{2}^{2}\left(124 \omega_{1}^{4}-696 \omega_{1}^{2}+81\right)}{\left(2 \omega_{1}^{2}-1\right)^{2}\left(5 \omega_{1}^{2}-1\right)} r_{1}^{2}+ \\
& +\frac{1}{6} \frac{\omega_{1} \omega_{2}\left(64 \omega_{1}^{4}-64 \omega_{1}^{2}-43\right)}{\left(2 \omega_{1}^{2}-1\right)^{2}\left(5 \omega_{1}^{2}-4\right)\left(5 \omega_{1}^{2}-1\right)} r_{1} r_{2}+ \\
& +\frac{1}{144} \frac{\omega_{1}^{2}\left(124 \omega_{1}^{4}+448 \omega_{1}^{2}-491\right)}{\left(2 \omega_{1}^{2}-1\right)^{2}\left(5 \omega_{1}^{2}-4\right)} r_{2}^{2} .
\end{aligned}
$$

The variables $r_{1}, r_{2}$ are introduced by formulae (6) . The discriminant $D_{4} \equiv$ $K_{4}\left(r_{1}=\omega_{2}, r_{2}=\omega_{1}\right)=c_{20} \omega_{2}^{2}+c_{11} \omega_{1} \omega_{2}+c_{02} \omega_{1}^{2}$ is then

$$
D_{4}=\frac{644 \gamma^{4}-541 \gamma^{2}+36}{16\left(4 \gamma^{2}-1\right)\left(25 \gamma^{2}-4\right)},
$$

where $\gamma=\omega_{1} \omega_{2}$. This expression is identical to the formula obtained by Deprit and Deprit-Bartholomé [4].

In the next steps we obtain the normal forms of the 5th and 6th orders. According to formula (7), the general expression for the 6th order homogeneous component $K_{6}$ of the normal form $K^{(6)}$ is

$$
K_{6}=c_{30} r_{1}^{3}+c_{21} r_{1}^{2} r_{2}+c_{12} r_{1} r_{2}^{2}+c_{03} r_{2}^{3} .
$$

In view of immense computer memory consumption in the calculations, the final expressions have been computed individually for each term of the normal form. The peak memory load during the computation of the terms with the coefficients $c_{30}$ and $c_{03}$ (the terms which are "homogeneous" with respect to the kind of the variables) reached $\approx 0.9$ GByte, and in the case of the "mixed" terms (those with the coefficients $c_{21}$ and $c_{12}$ ) it reached $\approx 1.3$ GByte. However, the resulting coefficients are quite compact: 


$$
\begin{aligned}
c_{30}= & -1 / 62208 / \omega_{1} \cdot\left(349789120 \omega_{1}^{18}-1262731648 \omega_{1}^{16}+2425101616 \omega_{1}^{14}-\right. \\
& -3030520672 \omega_{1}^{12}+2222006908 \omega_{1}^{10}-882757372 \omega_{1}^{8}+207906387 \omega_{1}^{6}- \\
& \left.-31372317 \omega_{1}^{4}+2661813 \omega_{1}^{2}-83835\right) / \\
& /\left(2 \omega_{1}^{2}-1\right)^{5} /\left(10 \omega_{1}^{2}-1\right) /\left(5 \omega_{1}^{2}-1\right)^{3}, \\
c_{21}= & -1 / 1728 \cdot\left(3176280000 \omega_{1}^{24}-19106816000 \omega_{1}^{22}+63106722600 \omega_{1}^{20}-\right. \\
& -141142031300 \omega_{1}^{18}+213727654214 \omega_{1}^{16}-215397500295 \omega_{1}^{14}+ \\
& +143749752195 \omega_{1}^{12}-63768859339 \omega_{1}^{10}+19069932231 \omega_{1}^{8}- \\
& \left.-3889018750 \omega_{1}^{6}+505561132 \omega_{1}^{4}-32077584 \omega_{1}^{2}+400896\right) / \\
& /\left(2 \omega_{1}^{2}-1\right)^{5} / \omega_{2} /\left(10 \omega_{1}^{2}-1\right) /\left(5 \omega_{1}^{2}-4\right)^{3} /\left(5 \omega_{1}^{2}-1\right)^{3}, \\
& 1 / 1728 \cdot \omega_{1} \cdot\left(3176280000 \omega_{1}^{22}-19008544000 \omega_{1}^{20}+62566226600 \omega_{1}^{18}-\right. \\
& -137831914700 \omega_{1}^{16}+202885849514 \omega_{1}^{14}-196481798617 \omega_{1}^{12}+ \\
& +124356412922 \omega_{1}^{10}-51393703020 \omega_{1}^{8}+14020325316 \omega_{1}^{6}- \\
& \left.-2566329143 \omega_{1}^{4}+291589800 \omega_{1}^{2}-13993776\right) / \\
& /\left(2 \omega_{1}^{2}-1\right)^{5} /\left(10 \omega_{1}^{2}-9\right) /\left(5 \omega_{1}^{2}-1\right)^{3} /\left(5 \omega_{1}^{2}-4\right)^{3}, \\
c_{03}= & 1 / 62208 \cdot \omega_{1}^{2} \cdot\left(349789120 \omega_{1}^{16}-1885370432 \omega_{1}^{14}+4915656752 \omega_{1}^{12}-\right. \\
& -7970990576 \omega_{1}^{10}+8326473644 \omega_{1}^{8}-5330237408 \omega_{1}^{6}+1834402891 \omega_{1}^{4}- \\
& \left.-221117724 \omega_{1}^{2}-18522432\right) / \\
& /\left(2 \omega_{1}^{2}-1\right)^{5} / \omega_{2} /\left(10 \omega_{1}^{2}-9\right) /\left(5 \omega_{1}^{2}-4\right)^{3} .
\end{aligned}
$$

Substituting for the values of the coefficients in the discriminant $D_{6} \equiv$ $K_{6}\left(r_{1}=\omega_{2}, r_{2}=\omega_{1}\right)=c_{30} \omega_{2}^{3}+c_{21} \omega_{1} \omega_{2}^{2}+c_{12} \omega_{1}^{2} \omega_{2}+c_{03} \omega_{1}^{3}$, one has the Deprit formula analogue in the 6 th order of normalization:

$$
\begin{aligned}
D_{6}= & 1 / 20736 \cdot\left(-16096320+578209968 \gamma^{2}-5879019660 \gamma^{4}+\right. \\
& +23361243081 \gamma^{6}-32843706320 \gamma^{8}-104264873152 \gamma^{10}+ \\
& \left.+481275622400 \gamma^{12}+94280800000 \gamma^{14}\right) / \\
& / \gamma /\left(4 \gamma^{2}-1\right)^{5 / 2} /\left(25 \gamma^{2}-4\right)^{3} /\left(100 \gamma^{2}-9\right)
\end{aligned}
$$

This expression is in agreement with that obtained for $D_{6}$ by Meyer and Schmidt [10] and Schmidt [11].

Now consider a particular case of the planar circular RTBP, namely, the case when the ratio of masses of the main gravitating bodies $\mu=\mu_{3}$. The 
corresponding value of $\gamma$ is found by equating the numerator of the Deprit formula (12) to zero and solving the quadratic equation. This gives

$$
\gamma=\frac{\left(541-199945^{1 / 2}\right)^{1 / 2}}{2 \cdot 322^{1 / 2}}=0.269931985621 \ldots
$$

Then, discriminant (18) in exact numeric representation simplifies to

$$
D_{6}=-\frac{5 \cdot 2^{1 / 2}\left(15711930947857+41876715371 \cdot 199945^{1 / 2}\right)\left(253+199945^{1 / 2}\right)^{1 / 2}}{96703113019392},
$$

and in numeric form with fixed precision

$$
D_{6}=-66.6297952504 \ldots
$$

The obtained floating-point numerical value confirm the results by Markeev [5, 6, 8] and Coppola and Rand [9], while the obtained exact numeric value (20) confirms the result by Schmidt [11]. Schmidt [11] gives this value in a somewhat different algebraic representation.

The frequencies and the mass ratio itself in the $\mu=\mu_{3}$ case are evaluated to

$$
\begin{aligned}
\omega_{1} & =1 / 644 \cdot\left(\left(5^{1 / 2} \cdot 39989^{1 / 2}-219\right)^{1 / 2}+322^{1 / 2}\right)^{1 / 2} \cdot 2^{1 / 2} \cdot 322^{3 / 4}= \\
& =0.959622914235 \ldots, \\
\omega_{2} & =1 / 644 \cdot\left(-\left(5^{1 / 2} \cdot 39989^{1 / 2}-219\right)^{1 / 2}+322^{1 / 2}\right)^{1 / 2} \cdot 2^{1 / 2} \cdot 322^{3 / 4}= \\
& =0.281289641605 \ldots, \\
\mu_{3} & =\frac{1}{2}-\frac{1}{6}\left(\frac{3265+2 \cdot 199945^{1 / 2}}{483}\right)^{1 / 2}=0.0109136676772 \ldots
\end{aligned}
$$

We substitute the frequencies in the coefficients $c_{30}, c_{21}, c_{12}, c_{03}$, and evaluate the coefficients in numeric form with fixed precision:

$$
\begin{aligned}
& c_{30}=-0.219259187025 \ldots, \\
& c_{21}=7.79324843205 \ldots, \\
& c_{12}=-209.933620500 \ldots, \\
& c_{03}=-14.5264460461 \ldots
\end{aligned}
$$


The obtained floating-point numeric values for the normal form coefficients are in agreement with the floating-point computations by Markeev [5, 6, 8] to 3-5 significant digits, and with the floating-point computations, performed much later on by Coppola and Rand [9], to 9 significant digits, i.e., to the accuracy with which Coppola and Rand [9] stated their results.

Note that the exact numeric value of $D_{6}$, given by formula (201), is evidently nonzero; hence, Markeev's theorem [5, 8] can be straightforwardly applied to infer that the motion is stable. Evaluation of (20) to a number with fixed precision is not necessary. It is interesting that the big number 41876715371 in formula (20) is prime, and 15711930947857 factors only in two prime numbers, 2317052197 and 6781.

Intermediary analytical expressions in the procedure of nonlinear normalization occupy gigabytes of the main memory, but the final expressions, Eqs. (14) -(18) and (20), are compact enough to be presented in typographic format. Of course, there exist ways of derivation of these formulae with less memory consumption. On one hand, this can be done by utilizing specific individual properties of the problem in constructing specialized algebraic processors, as accomplished in [10, 11]. On the other hand, this problem represents a promising field of application for the so-called method of numeric deduction of analytical expressions, described in [27, 28]. In brief, the method consists in exact numeric calculation of a derived expression on a set of simple (rational) values of the parameters and in subsequent "restoration" of the expression.

\section{Conclusions}

The problem of stability of the triangular libration points in the planar circular restricted three-body problem has been considered. A software package, intended for normalization of autonomous Hamiltonian systems by means of computer algebra, has been designed in the Maple language and has been used to obtain normal forms of the Hamiltonian.

The normalization has been carried out up to the 6th order of expansion of the Hamiltonian in the coordinates and momenta. Analytical expressions for the coefficients of the Birkhoff normal form have been derived. Though intermediary expressions occupy gigabytes of the computer memory, the obtained coefficients of the normal form are compact enough for presentation in the typographic format (Eqs. (14)-(17) ). The analogue of the Deprit formula 
for the stability criterion has been derived in the 6th order of normalization (Eq. (18)). The obtained floating-point numerical values for the normal form coefficients and the stability criterion confirm the results by Markeev [5, 6,, 8$]$ and Coppola and Rand [9], while the obtained analytical and exact numeric expressions for the stability criterion in the 6th order of normalization confirm the results by Meyer and Schmidt [10] and Schmidt [11].

It is important that the given computational problem has been solved without constructing a specialized algebraic processor; i.e., the designed computer algebra package has a broad field of applicability.

The author is thankful to A.D. Bruno for valuable remarks. This work was partially supported by the Russian Foundation for Basic Research (project \# 05-02-17555) and by the Programme of Fundamental Research of the Russian Academy of Sciences "Fundamental Problems in Nonlinear Dynamics".

\section{References}

[1] J.J. Lagrange, Essai sur le problème des trois corps (1772), Ouvres de Lagrange, v. 6, pp. 272-282 (Gauthier-Villars, Paris, 1873).

[2] G. Gascheau, Examen d'une classe d'équations différentielles et application à un cas particulier du problème des trois corps, Comptes Rendus 16 (1843) 393-394.

[3] A.M. Leontovich, On the stability of the Lagrange periodic solutions of the restricted three-body problem, Doklady Akademii Nauk SSSR 143 (1962) 525-529. (In Russian.)

[4] A. Deprit, A. Deprit-Bartholomé, Stability of the triangular Lagrangian points, Astron. J. 72 (1967) 173-179.

[5] A.P. Markeev, On the stability of the triangular libration points in the circular restricted three-body problem, Prikl. Mat. Mekh. 33 (1969) 112116 (Russian); English translation: J. Appl. Math. Mech. 33 (1969) $105-110$.

[6] A.P. Markeev, Stability of the triangular Lagrangian solutions of the restricted three-body problem in the three-dimensional circular case, Astron. Zh. 48 (1971) 862-868 (Russian); English translation: Soviet Astronomy - AJ 15 (1972) 682-686. 
[7] A.G. Sokolsky, Stability of the Lagrange solutions of the restricted threebody problem for the critical ratio of the masses, Prikl. Mat. Mekh. 39 (1975) 366-369 (Russian); English translation: J. Appl. Math. Mech. 39 (1975) 342-345.

[8] A.P. Markeev, Libration Points in Celestial Mechanics and Cosmodynamics, Nauka Publishers, Moscow, 1978. (In Russian.)

[9] V.T. Coppola, R.H. Rand, Computer algebra implementation of Lie transforms for Hamiltonian systems: Application to the nonlinear stability of $L_{4}$, ZAMM - Journal of Applied Mathematics and Mechanics 69 (1989) 275-284.

[10] K.R. Meyer, D.S. Schmidt, The stability of the Lagrange triangular point and a theorem of Arnol'd, Journal of Differential Equations 62 (1986) 222-236.

[11] D.S. Schmidt, The stability of the Lagrangian point $L_{4}$, Celest. Mech. 45 (1989) 201-206.

[12] A.G. Sokolsky, I.I. Shevchenko, Nonlinear normalization of autonomous Hamiltonian systems on computers in analytical form, Preprint of the Inst. of Theoretical Astronomy of the USSR Acad. Sci., No. 8 (1990) 51 pp. (In Russian.)

[13] I.I. Shevchenko, A.G. Sokolsky, Algorithms for normalization of Hamiltonian systems by means of computer algebra, Computer Physics Communications 77 (1993) 11-18.

[14] D.S. Schmidt, Versal normal form of the Hamiltonian function of the restricted problem of three bodies near $L_{4}$, J. Comput. Appl. Math. 52 (1994) 155-176.

[15] A.D. Bruno, A.G. Petrov, On calculation of the Hamiltonian normal form, Doklady Akademii Nauk 410 (2006) 474-478 (Russian); English translation: Doklady Physics 51 (2006) 555-559.

[16] B.W. Char, K.O. Geddes, G.H. Gonnet, B.L. Leong, M.B. Monagan, S.M. Watt, Maple V Library Reference Manual, Springer, New York, 1993. 
[17] V.I. Arnold, Mathematical Methods of Classical Mechanics, Springer, New York, 1980.

[18] A.D. Bruno, The Restricted Three-Body Problem: Plane Periodic Orbits, Walter de Gruyter, Berlin, 1994.

[19] A.P. Markeev, S.V. Medvedev, A.G. Sokolsky, Methods and Algorithms for Normalization of Differential Equations, Moscow Aviation Inst., Moscow, 1985. (In Russian.)

[20] A.G. Sokolsky, I.I. Shevchenko, On normalization of autonomous Hamiltonian systems on computers in analytical form, Preprint of the Inst. of Theoretical Astronomy of the USSR Acad. Sci., No. 14 (1991) 72 pp. (In Russian.)

[21] A.C. Hearn, REDUCE User's Manual, Version 3.2, The Rand Corporation, Santa Monica, 1985.

[22] A. Deprit, Canonical transformations depending on a small parameter, Celest. Mech. 1 (1969) 12-30.

[23] G.I. Hori, Theory of general perturbations with unspecified canonical variables, Journ. of Japan Astron. Soc. 18 (1966) 287-296.

[24] W.A. Mersman, A new algorithm for the Lie transformation, Celest. Mech. 3 (1970) 81-89.

[25] N.A. Sushko, I.I. Shevchenko, "NF" - a package of routines for normalization of Hamiltonian systems, in: Abstracts of the Conference "Problems of Celestial Mechanics", Inst. of Theoretical Astronomy of the Russian Acad. Sci., St.Petersburg, 1997, pp. 166-168. (In Russian.)

[26] D.A. Sushko, I.I. Shevchenko, Librations of a nonsymmetric satellite about its center of mass in a circular orbit, Kosmich. Issled. 34 (1996) 300-311 (Russian); English translation: Cosmic Research 34 (1996) 277288.

[27] I.I. Shevchenko, N.N. Vasiliev, Algorithms of numeric deduction of analytical expressions, SIGSAM Bulletin 27, No. 1 (1993) 1-3. 
[28] I.I. Shevchenko, Numeric deduction in symbolic computation. Application to normalizing transformations, Journal of Symbolic Computation 24 (1997) 103-111. 